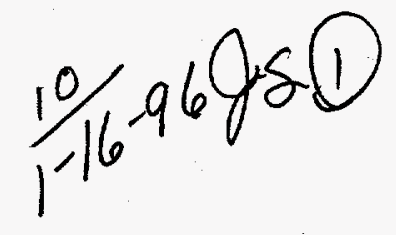

SLAC-PUB-95-6627

SLAC/SSRL-00090

LBL-36027

October 1995

\title{
Performance of the Elliptically Polarizing Undulator on SPEAR
}

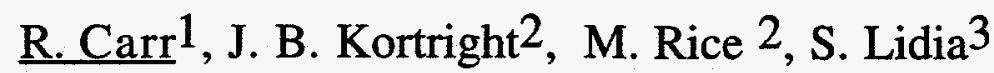

1) Stanford Linear Accelerator Center, Stanford Synchrotron Radiation Laboratory, Stanford California 94309, USA

2) Center for X-Ray Optics, Lawrence Berkeley Laboratory, Berkeley, CA 94720

3) Physics Department, University of California, Davis CA 95616

\section{$\underline{\text { Abstract }}$}

This is a description of the first tests of the Elliptically Polarizing Undulator (EPU) on the SPEAR storage ring at SSRL. The EPU is the first device of its type; it is capable of producing plane polarized light in the vertical and horizontal, and right and left circularly polarized light in the $500-1000 \mathrm{eV}$ range. Tests of the EPU were done to characterize its effect on the electron beam in SPEAR. Even at minimum gap, motion of the EPU magnets to vary the polarization of the output radiation caused negligible changes in the tune or the steering of the electron beam, even with no compensation of the steering trim coils. We also measured the polarization of $x$-rays generated by the EPU using a newly developed multilayer polarimeter built to be efficient in the EPU's energy range. The EPU produces nearly $100 \%$ plane and circularly polarized $x$-rays. Using left and right circularly polarized radiation, we also performed tests of magnetic circular dichroism on magnetic multilayers.

DISTRIBUTION OF THIS DOCUMENT IS UNLIMITED

\section{Introduction}

Magnetic and biological materials that exhibit circular dichroism in $\mathrm{x}$-ray absorption are currently of great research interest and frequently contain metals in the first row transition series. These metals absorb at L-edges in the $500-1000 \mathrm{eV}$ range. Between 300 and $3000 \mathrm{eV}$, there are no quarter wave plates presently available to 


\section{DISCLAIMER}

Portions of this document may be illegible in electronic image products. Images are produced from the best available original document. 
create circularly polarized light. Fortunately, in this energy range, grazing incidence monochromator optics do not affect polarization significantly.

Bending magnet sources of circularly polarized radiation have low flux, require separate optical paths for left and right circularly polarized radiation, and are not useful at very high polarization rates. To obtain a higher flux and polarization, various insertion devices have been designed, including several undulators. [1] Of these approaches, we were attracted to the single helical undulator for simplicity and high flux. The result is the elliptically polarizing undulator (EPU). [2] Our design built on a magnet arrangement of Sasaki [3] and the phase tunability concept of our adjustable phase undulator [4] This design, shown in figure 1, comprises two planes of magnets, one above and one below the storage ring's electron beampipe. Each plane consists of two rows of pure $\mathrm{NdFeB}$ magnet blocks in the Halbach sinusoidal arrangement [5]. Each of the rows is mounted on slides, and can be moved longitudinally parallel to the electron beam, by $\pm 1 / 2$ the undulator period length, $\lambda$. The EPU is mounted on the SPEAR beamline 5 multiundulator mover; it is one of 5 undulators that can be positioned over the beampipe. The mover can vary the gap between 30 and $200 \mathrm{~mm}$. The EPU has 26 periods; each period is $65 \mathrm{~mm}$ long and it generates $\mathrm{X}$-rays in the $500-1000 \mathrm{eV}$ range when SPEAR is operated at $3 \mathrm{GeV}$.

\section{Performance Results}

We consider three sets of motions for the magnet rows in each quadrant: Row Phase $(\phi \rho)$ (upper left and lower right move one way while upper right and lower left move the other), Jaw Phase (upper left and right one way, lower left and right the other), and Pair Phase (upper and lower left one way, upper and lower right the other). The jaw phase or pair phase motions can be used to tune the strength of the magnetic field and thus the energy of the $\mathrm{x}$-rays produced. Variation of $\phi \rho$ changes the polarization of $x$-rays. We chose a zero of motion where the magnetic field on-axis is horizontal, so the $\mathrm{x}$-rays are plane polarized vertically. At about 
$\phi_{\rho}= \pm 0.16 \lambda$, depending slightly on the gap, the magnetic field is a circular helix, so the x-rays are left or right circularly polarized. At $\phi_{\rho}= \pm 0.5 \lambda$ the magnetic field is vertical, and the $x$-rays are plane polarized horizontally, as in a conventional undulator.

We find that the EPU phase changes are transparent to SPEAR; neither row, jaw, pair or uncoordinated phase motions have any noticeable effect on beam position monitors around the ring, and users at other beamlines see no effects. Therefore, we expect to be able to move the EPU between right and left circular polarization modes at frequent intervals; the mechanical time required is about 5 seconds.

Because of SPEAR's large emittance (129 nm-rad horizontal, $3 \mathrm{~nm}$ rad vertical) the width of the EPU fundamental is much larger than $1 / \mathrm{N}=1 / 26$, and we also noticed a broad second harmonic peak. The second harmonic peak was examined by polarimetry, and found to be strongly polarized in the same sense as the fundamental. Beam position monitor measurements showed that the beam did not pass exactly along the EPU axis. We moved the undulator transversely to minimize the energy of the fundamental, but even then, the beam moved at an angle to the axis both vertically and horizontally. This degrades polarization only a little. However, the EPU was designed so that we could also change the energy of the $x$-rays, at any polarization, by changing the jaw or pair phases. Because the beam was not on axis, we found that polarization was not well maintained when the jaw or pair phase was changed.

The beamline 5 monochromator reflects the $x$-ray beam from five platinum surfaces. A two degree grating was used in this energy range, and this is the maximum incidence angle for any reflection. Under these conditions, the monochromator is not expected to affect the polarization of the light passing through it.

A simple multilayer polarimeter was used to characterize the polarization of EPU beams as the row phase was varied. The polarimeter comprised a rotatable linear polarizer in the form of a 
multilayer reflector set at $45^{\circ}$, near the Brewster angle [6]. A sputtered $\mathrm{W} / \mathrm{B}_{4} \mathrm{C}$ multilayer with $\mathrm{d}=12.43 \AA$ and 75 periods was used, yielding a Bragg peak at $708 \mathrm{eV}$. The extinction ratio $R_{S} / R_{p}$ $\approx 3 \times 10^{4}$ is quite high, and even though $R_{S} \approx 0.01$, this reflector acts as an excellent linear polarizer at $700 \mathrm{eV}$. About 1010 photons/second enter the polarimeter through its entrance pinhole. This is the highest photon energy at which multilayers have been used as polarizers to date, and the results demonstrate that multilayers can be useful for polarimetry at the 3-d transition metal L edges. A semitransparent mesh monitored the incident intensity and a silicon diode detector received the beam reflected from the multilayer.

A set of polarimetry scans for a variety of row phase settings ranging from vertical linear to circular to horizontal linear polarization are shown in figure 2. Each scan shows the intensity reflected by the polarizer as it rotates $360^{\circ}$ about the beam direction. Zero on the azimuthal angle scale corresponds to an upward vertical reflection. The scans show the expected polarization behavior ranging from predominantly vertical linear at $\phi_{\rho} \approx 0$, to circular at $\phi_{\rho} \approx 0.16 \lambda$, to horizontal linear at $\phi_{\rho} \approx 0.5 \lambda$. The changing intensities of the different scans results predominantly from the changing energy of the undulator first harmonic as $\phi \rho$, and hence magnetic field strength, change. Thus the measurement energy of $708 \mathrm{eV}$ falls at different positions on the broad undulator peak as $\phi \rho$ changes. The energy shift of the first harmonic was so strongly dependent on $\phi_{\rho}$, that in order to remain on the first peak over its full range, the undulator gap was changed from $35 \mathrm{~mm}$ (for $\phi \rho \leq$ $0.2 \lambda$ ) to $42 \mathrm{~mm}$ (for $\phi \rho \geq 0.2 \lambda$ ).

The polarimetry data were analyzed to determine the Stokes parameters of the beam at each setting. Following conventions for rotating analyzer polarimetry [7], the individual scan intensities were fit to:

$$
I(\alpha)=\frac{1}{2}\left(R_{S}+R_{p}\right) S_{0}+\frac{1}{2}\left(R_{s}-R_{p}\right)\left[S_{1} \cos (2 \alpha)+S_{2} \sin (2 \alpha)\right]
$$


where $\alpha$ is the azimuthal angle of the polarizer and the Stokes parameters are $S_{0}, S_{1}, S_{2}$, and $S_{3}$. Each scan was fit to obtain values for $S_{0}, S_{1}$, and $S_{2}$ which were normalized by $S_{0}$. Using just a polarizer, we can determine $S_{1}$, and $S_{2}$, but not $S_{3}$, because we cannot distinguish between the circularly polarized and unpolarized fractions. To set an upper limit on possible unpolarized radiation, we identified the $\phi \rho$ value $(0.48 \lambda)$ yielding the highest degree of linear polarization $P_{L}=\sqrt{\left(S_{1}^{2}+S_{2}^{2}\right)} / S_{0}=0.98$, and then assumed that all remaining intensity was unpolarized radiation. For calculations, we used the same degree of total polarization $P_{L}=\sqrt{\left(S_{1}^{2}+S_{2}^{2}+S_{3}^{2}\right)} / S_{0}=0.98$ for all $\phi_{\rho}$. Then the value of $S_{3}$ representing circular polarization was calculated from this last expression with $S_{1}$ and $S_{2}$ measured and $P$ assumed the same for each. As seen in figure 3, when radiation does not come from the peak of the undulator fundamental, it has a linearly polarized and an unpolarized component. The unpolarized component comes from off-axis electrons and off-axis photons. These effects account for the unpolarized radiation we observed.

The variation obtained in Stokes parameters with $\phi \rho$ is shown in figure 4. $S_{2}$ represents linear polarization at $\pm 45^{\circ}$, and is negligible for all $\phi_{\rho}$. The largest amount of vertical linear polarization $S_{1}$ was -0.90 at $\phi_{\rho}=0$, while the largest amount of horizontal linear polarization was 0.98 at $\phi \rho=0.48 \lambda$. The circular polarization shows a broad peak near $\phi_{\rho}=0.16 \lambda$ with maximum of $S_{3}=0.98$. These degrees of linear and circular polarization at their optimum row phase settings are very high, and could be improved with better beam steering. They would also improve if the undulator fundamental peak were shifted to $708 \mathrm{eV}$ at each $\phi \rho$ setting.

To confirm that both senses of circular polarization are produced at the appropriate settings, magnetic circular dichrosim data were collected from a $\mathrm{Fe} / \mathrm{Cr}$ multilayer. The multilayer consisted of 30 periods of nominally $41 \AA \mathrm{Fe}$ and $7 \AA \mathrm{Cr}$ deposited by magnetron sputtering onto a silicon wafer substrate. MCD data were obtained using total yield with the sample remnantly magnetized in-plane after magnetizing between the poles of a $1 \mathrm{kG}$ electromagnet, and 
are shown in figure 5. X-rays struck the multilayer at a glancing angle of about $10^{\circ}$. The distinct MCD signal at the Fe LII and LIII edges shows the opposite helicity of circular polarization for $\phi \rho= \pm$ $0.16 \lambda$, which cannot be verified using the linear polarizer alone.

\section{Summary}

The EPU was tested and shown to produce negligible interference with the SPEAR electron beam when its rows of magnets were moved. The row phase motion was shown to produce linearly and circularly polarized light, as expected from theory. The degree of polarization was almost $100 \%$, in spite of electron beam missteering. We have also demonstrated the first use of multilayers as polarizers in this energy range. We expect the EPU to be a very useful source for magnetic circular dichroism studies.

\section{Acknowledgments}

This research was performed at SSRL which is operated by the Department of Energy, Office of Basic Energy Sciences. The authors thank F. Coffman of SSRL for his timely assistance with the data acquisition system. J.K and M.R. were supported by the Office of Basic Energy Science of the U.S. Department of Energy under contract DE03-76SF00098. M. R. was also supported by the University of California President's Postdoctoral Fellowship Program.

References

[1] K-J. Kim, SPIE Proceedings 1345, p. 116 (1990)

[2] R. Carr and S. Lidia, Proceedings of the 1993 SPIE Conference on Electron Beam Sources of High Brightness Radiation \#2013.

[3] S. Sasaki, K. Miyata, and T. Takada, Jpn. J. Appl. Phys. 31, p. L1794 (1992)

[4] R. Carr, Nucl. Inst. \& Methods A306, p. 391, (1991)

[5] K. Halbach, Nucl. Inst. \& Methods 187, p. 109 (1981)

[6] J.B. Kortright, M. Rice, and K.D. Frank, these proceedings 
[7] E. Collett, "Polarized Light, Fundamentals and Applications," (Marcel Dekker, Inc., New York, 1993), Ch. 4 and 6.

\section{Figure Captions}

Figure 1: Schematic concept drawing of the EPU. The four rows of magnets are movable independently.

Figure 2. Polarimeter scans show strong dependence of polarization state on row phase setting. Vertical linear polarization produces maxima at $0^{\circ}$ and $180^{\circ}$, while horizontal linear polarization produces maxima at $\pm 90^{\circ}$. Circular polarization produces the flattest scans.

Fig 3: Calculated flux curve for the EPU, using SPEAR's emittance, that shows various rates of polarization. The unpolarized component is due to off-axis radiation and finite emittance of the electron beam. The row phase is set to produce $100 \%$ circular polarization at the undulator first harmonic peak. The effects beam missteering are not included.

Figure 4. Stokes parameters as a function of row phase setting, showing the inverse relationship between linear and circular polarization states.

Figure 5. Magnetic circular dichrosim is observed in a remnantly magnetized $\mathrm{Fe} / \mathrm{Cr}$ multilayer. The top two scans show the total yield spectra taken with $S_{3} \geq \pm 0.98$, and the bottom curve is the MCD signal, $\left(\mathrm{I}_{+}-\mathrm{I}_{-}\right) /\left(\mathrm{I}_{+}+\mathrm{I}_{-}\right)$.

\section{DISCLAIMER}

This report was prepared as an account of work sponsored by an agency of the United States Government. Neither the United States Government nor any agency thereof, nor any of their employees, makes any warranty, express or implied, or assumes any legal liability or responsibility for the accuracy, completeness, or usefulness of any information, apparatus, product, or process disclosed, or represents that its use would not infringe privately owned rights. Reference herein to any specific commercial product, process, or service by trade name, trademark, manufacturer, or otherwise does not necessarily constitute or imply its endorsement, recommendation, or favoring by the United States Government or any agency thereof. The views and opinions of authors expressed herein do not necessarily state or reflect those of the United States Government or any agency thereof. 


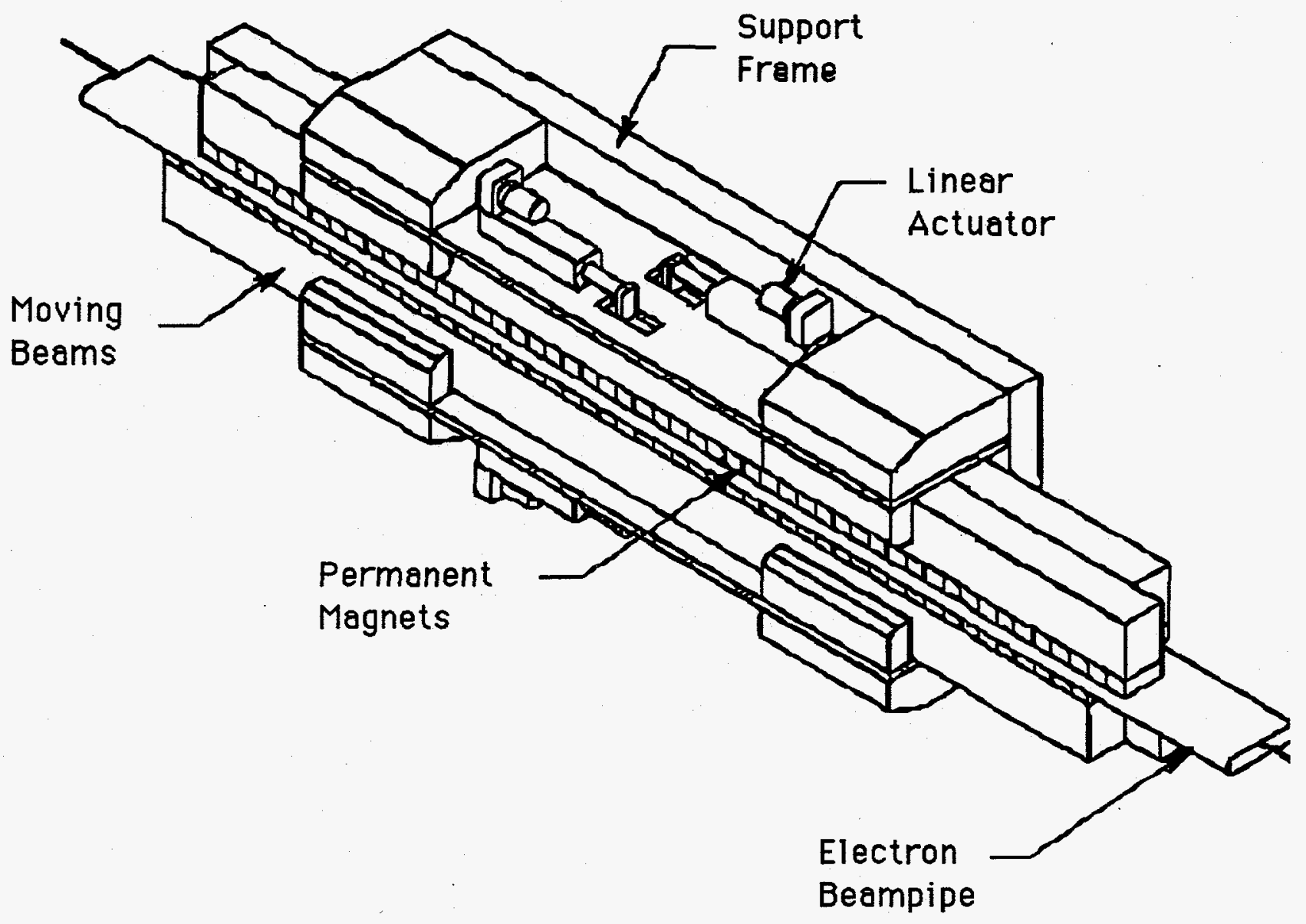

Figure 1, TUE6, Carr 


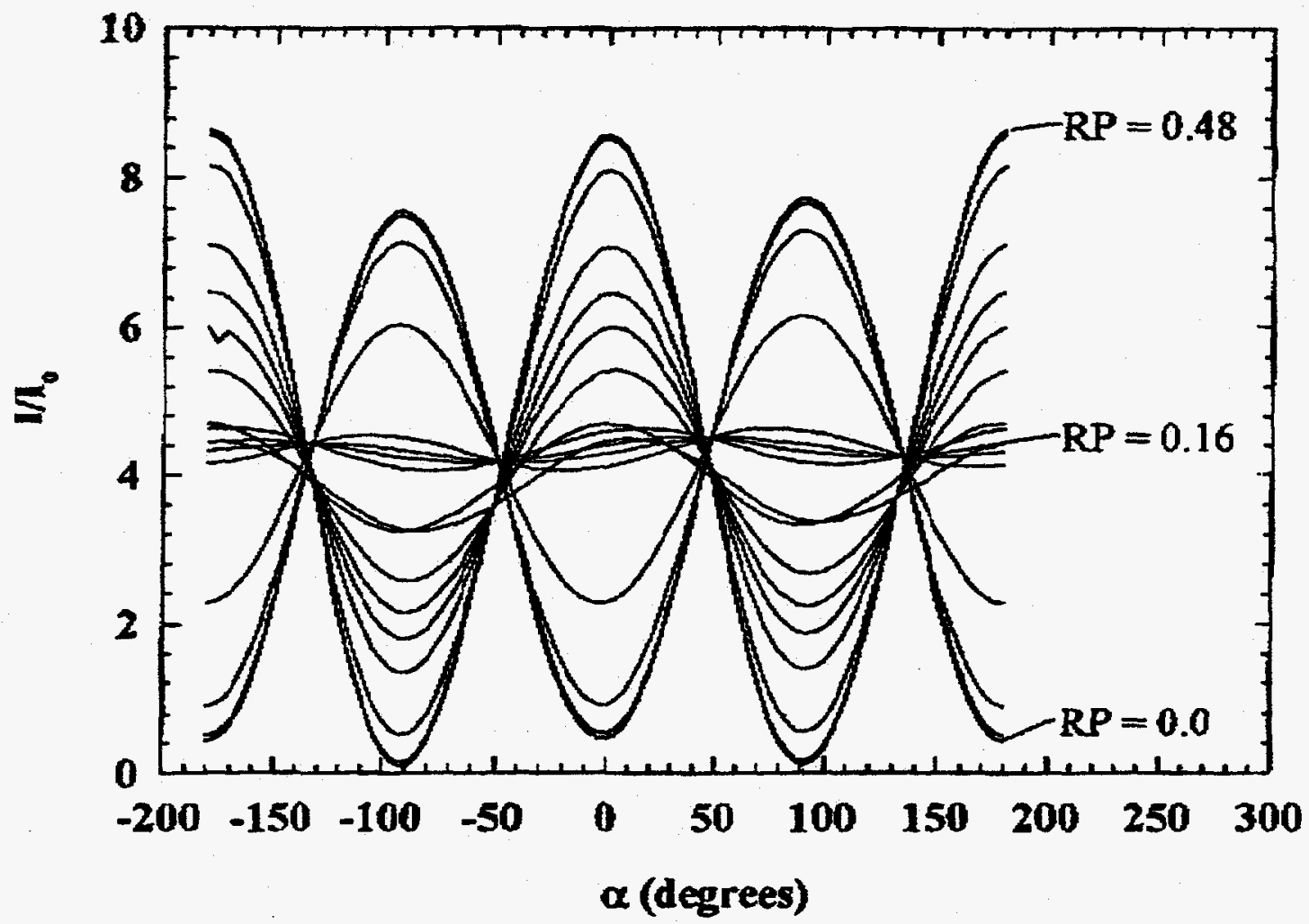

Figure 2, TUE6, Carr 


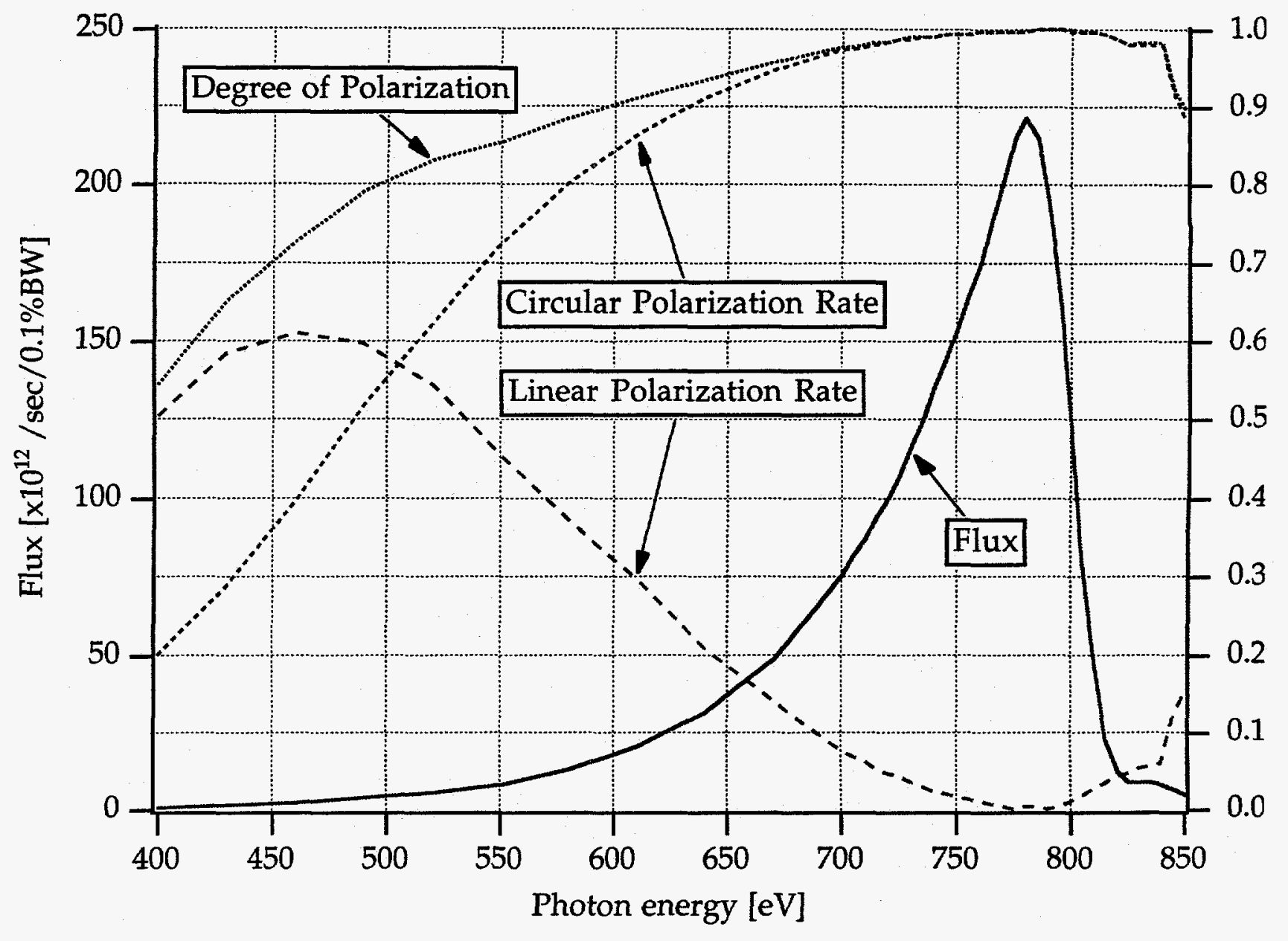

Figure 3, TUE6, Carr 


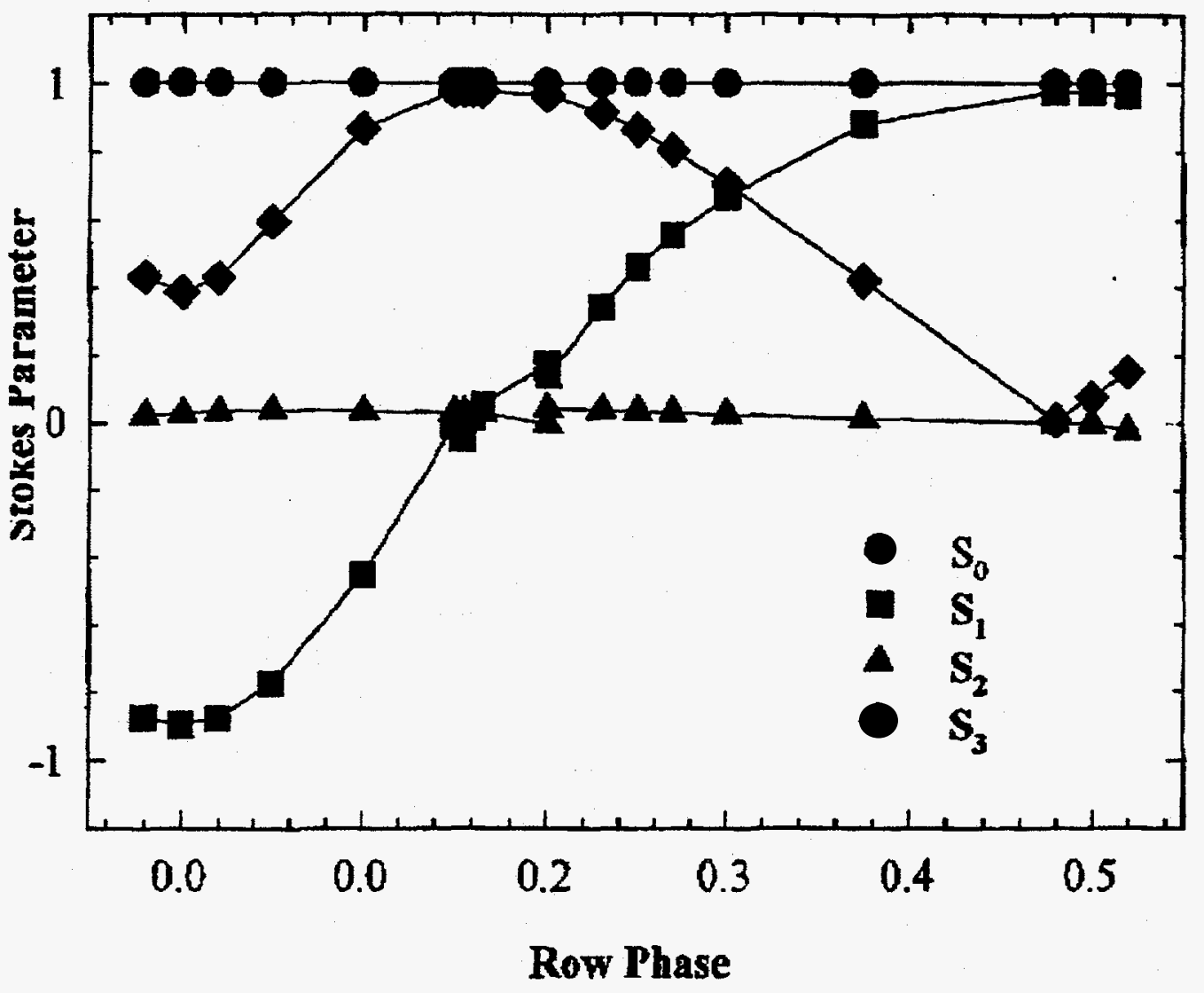

Figure 4, TUE6, Carr 


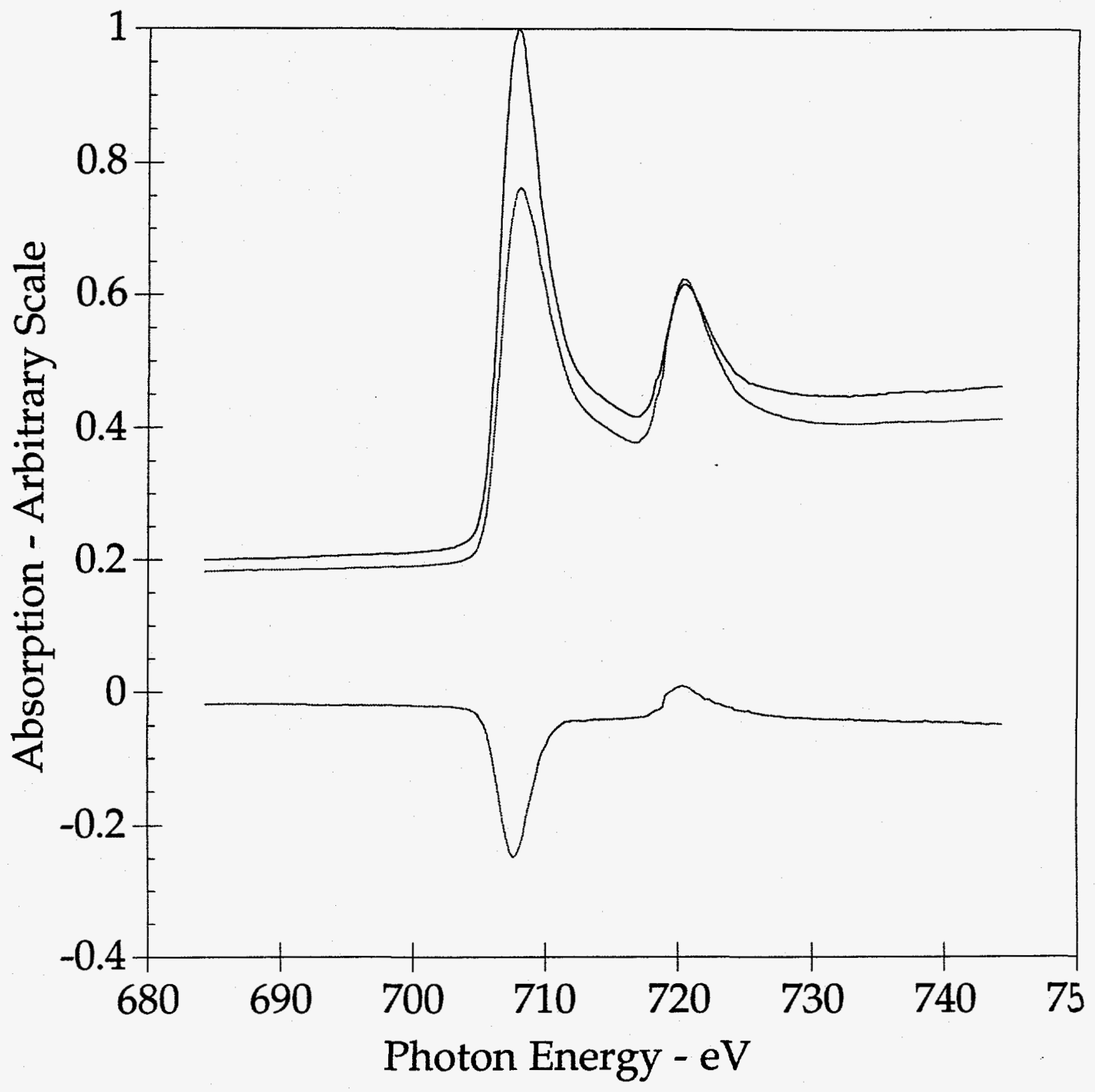

Figure 5, TUE6, Carr 